\title{
Africa Journal of Rheumatology: enhancing the visibility of rheumatology in Africa
}

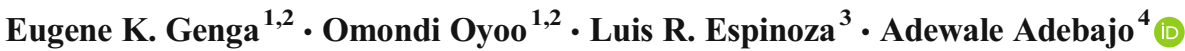

Received: 5 July 2017 / Accepted: 10 July 2017 / Published online: 27 July 2017

(C) International League of Associations for Rheumatology (ILAR) 2017

Clinical Rheumatology welcomes the African Journal of Rheumatology as an important development for the furtherance of rheumatological scholarship and education on the African continent and for rheumatology research in Africans. It is hoped that this development will in turn raise the profile of rheumatological conditions in Africa and among Africans. In particular, it is hoped that this will lead to the much needed collection of African musculoskeletal epidemiological and health services data, assist in the training of African rheumatologists, help to open up African rheumatology to the global rheumatology community, and ultimately improve the quality of care for myriads of Africans with rheumatic disorders.

The current population of Africa is 1,241,858,354 which is equivalent to $16.36 \%$ of the total world population based on the latest United Nations estimates [1]. There are many challenges facing Africa including limited financial resources, misuse of finances, malnutrition, poor water, and sanitation among others. Despite these many challenges faced in Africa, in recent times, the continent has undergone rapid economic growth and development. The available healthcare resources are overburdened by

Adewale Adebajo

a.o.adebajo@sheffield.ac.uk

1 Department of Clinical Medicine and Therapeutics, College of Health Sciences, University of Nairobi/Kenyatta National Hospital, Nairobi, Kenya

2 Nairobi Arthritis Clinic, Nairobi, Kenya

3 Louisiana State University School of Medicine, New Orleans, LA, USA

4 Faculty of Medicine, Dentistry and Health, University of Sheffield, Sheffield, UK the high burden of communicable diseases and the rising prevalence of non-communicable diseases. Rheumatic diseases are therefore not considered a high priority by the various African governments. Part of the reason for this is due to the limited epidemiological data on rheumatic diseases and their burden in Africa. Scientific journals play a central role in the dissemination of research results which will ultimately impact on policy change. Horton et al. [2] noted that researchers and policy makers in developing countries believe that the main way to solve problems of developing countries is by using information from Western research rather than using local data to solve regional problems. He, however, noted that in Africa "there is already a well-developed local information culture that needs support, not swamping," noting, moreover, the lack of African journals in MEDLINE [2]. Researchers in Africa and the developing world require access not only as readers but also as authors: for them to feel part of the global science community, they need not only to obtain information but also to be able to contribute to it and take part in the global discourse. The continent's resources are prioritized towards infectious diseases like HIV and malaria over the now increasing non-communicable diseases. Data on rheumatic diseases in Africa has been limited partly due to lack of infrastructure thus under diagnosis but also due to low scholarly output. Thus, the Africa Journal of Rheumatology was born. Since its inception 5 years ago, it has provided an uninterrupted forum through which medical practitioners and scientists from Africa and beyond can publish their rheumatology research. It has become a rich source of information about rheumatic disorders in the continent and a timely addition to our worldwide rheumatology community [3]. The journal has published various research articles on diseases once thought to be rare in Africa. They 
range from rheumatoid arthritis, lupus, gout, myositis to rheumatology in HIV. Research articles published in the journal shows rheumatoid arthritis, juvenile idiopathic arthritis, systemic lupus erythematosus, and antiphospholipid syndrome to be increasing in frequency in the indigenous populations of East, West, Central, and Southern Africa [4-7]. The HIV pandemic has changed the epidemiological spectrum of diseases in Africa. It has led to an increase in a variety of previously rarely seen conditions like spondyloarthropathies, fibromyalgia, pyomyositis, and scleroderma. Various scholars have shared their experiences in the journal [8-12]. The journal has also provided a forum through which scholars have been able to share their experiences in management of the rheumatic diseases with biologic therapy. The results have been similar to data from around the world $[13,14]$. Case reports of rare diseases and review articles have not been left out and have enriched the content of the journal bringing diversity in the articles published.

The visibility of the journal is hampered by the low scholarly output. This is in part due to severe limitations in the overall economic development and especially in research infrastructure. Researchers have limited access to funding for research as most African countries have no national agencies that are responsible for research. This is compounded by limitations in scientific writing, designing, and conducting research and in reporting the results. Partnership with international journals like the African Journal Partnership Project is welcome to bridge that gap by training African health researchers to improve the quality and visibility of their research and make the Africa journal of rheumatology a better resource for local researchers and policy makers [15].

This journal has become a site for exchange of knowledge of local rheumatic diseases, research, and debate and providing a forum through which international research can be made applicable to the African set-up. The Africa Journal of Rheumatology encourages international agencies, which conduct research in the region to support the journal through submission of research and subscription to the publication. It is our hope that this journal will provide a big step to bridge the big gaps in rheumatology in Africa.
Compliance with ethical standards

Disclosures None.

\section{References}

1. Worldometers (2017) (www.Worldometers.info)

2. Horton R (2000a) Development aid: manna or myth? Lancet 356: 1044-1045

3. Espinoza LR (2014) Welcoming an African asset: African Journal of Rheumatology. Afr J Rheumatol 2(2):47-48

4. Otieno FO, Moots RJ, Oyoo GO (2017) Rheumatoid arthritis in Kenya. Afr J Rheumatol 5(1):1-2

5. Akintayo RO, Aworinde OO, Olawumi HO, Yusuf IA (2016) Antiphospholipid syndrome in Africa: a review. Afr J Rheumatol 3(1):3-8

6. Genga EK, Otieno FO, Oyoo GO (2015) Clinical profiles of patients SLE in Nairobi. Afr J Rheumatol 3(2):62-66

7. Adelowo F (2013) Systemic lupus erythematosus: not a rare disease among black Africans. Afr J Rheumatol 1(2):46-47

8. Oyoo GO, Genga EK, Otieno FO, Omondi EA (2016) Clinical patterns of juvenile idiopathic arthritis: a single tertiary centre experience in Kenya Nairobi. Afr J Rheumatol 4(2):66-71

9. Venkat R, Jawad ASM, Chikanza IC (2014) Spontaneous resolution of a case of anti-retroviral treatment-naïve HIV-associated polymyositis. Afr J Rheumatol 2(2):78-84

10. Ilovi S, Oyoo G (2013) Characteristics of systemic sclerosis patients in Nairobi, Kenya: a retrospective study. Afr J Rheumatol 1(1):8-12

11. Malombe NM, Oyoo GO, Maritim MC, Kwasa J (2013) Prevalence of fibromyalgia in ambulatory HIV positive patients with musculoskeletal pain at Comprehensive Care Clinic, Kenyatta National Hospital. Afr J Rheumatol 1(2):70-75

12. Ouédraogo DD, Ouédraogo T, Kaboré F et al (2013) Prevalence of HIV infection among the patients with an avascular necrosis of the femoral head in Ouagadougou, Burkina Faso Ouédraogo. Afr J Rheumatol 1(2):57-60

13. Oyoo GO, Otieno FO, Mbuthia B, Omondi EA, Genga EK (2015) Experience with rituximab in patients with rheumatoid arthritis in Nairobi, Kenya. Afr J Rheumatol 3(1):17-21

14. Elhabbash B, Tarsin R (2017) Certolizumab effect in a cohort of 60 Libyan patients with rheumatic diseases. Afr J Rheumatol 5(1):19 23

15. Muula AS (2008) Medical journals and authorship in low-income countries. Croat Med J 49:681-683 